Acta Cryst. (2002). A58 (Supplement), C82

THEORETICAL AND PRACTICAL ASPECTS OF PHASING WITH RADIATION DAMAGE

D. Borek $^{1}$ W. Minor ${ }^{2}$ Z. Otwinowski ${ }^{1}$

${ }^{1}$ Department of Biochemistry, UT Southwestern Medical School, Dallas, TX, 75390-903 USA ${ }^{2}$ Department of Molecular Physiology and Biological Physics, University of Virginia, Charlottesville, VA, 22908-073

Traditionally one assumes there is no significant anomalous signal for the native data (Friedel symmetry) and changes other than the overall decay are ignored in the analysis. However, one may often use the deviations from these two approximations for phasing. The analysis of the deviation from Friedel symmetry results in the SAD method for structure solution, that has recently become practical for very well diffracting crystals or for ones that contain an heavy atom (calcium or heavier). It has been established that the exposure of a crystal to $\mathrm{x}$-rays produces defined changes in the protein structure such as the disruption of disulfide bonds, deamidation and decarboxylation. This information can be used for phasing if one can estimate the electron density of such changes.

Our goal is to solve protein structures from a single native data set. We expect that adding the analysis of $\mathrm{x}$-ray induced changes to SAD will allow solving the majority of native protein crystal structures. The practical application of this method requires the development of an optimal experimental protocol and improvement of the phasing analysis. The current methods are sub-optimal in their treatment of experimental errors that are about the same magnitude as the phase-sensitive signal. We are developing novel approaches to error-treatment and phasing using the combined SAD and radiation induced changes method and the results will be presented.

This work is supported by NIH grant GM 53163.

Keywords: SAD, RADIATION DAMAGE, PHASING

Acta Cryst. (2002). A58 (Supplement), C82

\section{MACROMOLECULAR STRUCTURE DETERMINATION BY THE SAD METHOD USING A COLORED URANYL COMPLEX} M.-L. Chesne ${ }^{1}$ O. Bertrand ${ }^{2}$ J. Vicat ${ }^{1}$ R. Kahn ${ }^{1}$

${ }^{1}$ Institut de Biologie Structurale J.-P. Ebel, Grenoble, France ${ }^{2}$ INSERM U 76, Paris, France

Uranyl salts are commonly used to prepare heavy-atom derivatives of protein crystals, but these derivatives exhibit usually numerous sites with low occupancies. A new derivative of tetragonal hen egg-white lysozyme crystals has been obtained with an uranyl complex of monoazo dye by soaking in a 2 mm solution of dark-purple monoazo uranyl complex. As the uranyl complex concentrates into the crystal the derivative crystal becomes dark opaque while the drop solution becomes colorless. Diffraction data were collected to a resolution of $1.7 \AA$ using cu radiation from a rotating anode with a high anomalous signal from uranium $\left(\mathrm{f}^{\prime}=16 \mathrm{e}\right.$ ). Three uranyl binding sites, different to those already found with uranyl nitrate, were found from the anomalous patterson map. The protein structure was solved de novo by the sad method using the programs mlphare and $\mathrm{dm}$. It was refined using the program cns. The refined uranium site occupancies are very high $(>0.9)$. In their equatorial plane each uranyl have the following ligands: one dye molecule, which is a tridentate ligand, one carboxylate oxygen from an aspartic acid of the protein and one water molecule. The planar dye molecule is held between two arginine residues making hydrophobic interactions. Furthermore, the stability of the complex is strengthened by a hydrogen bond between an arginine nitrogen and the dye sulfonate group. Colored derivative crystals indicate binding of the complex on the protein in the crystals. Trials with different proteins as well as with other monoazo dyes are in progress.

Keywords: URANYL COMPLEX MACROMOLECULAR CRYSTALLOGRAPHY SAD
Acta Cryst. (2002). A58 (Supplement), C82

IN-HOUSE PHASING WITH IODIDE SOAKS

G. Bunkoczi $^{1}$ J. E. Debreczeni ${ }^{1}$ J. Kaercher ${ }^{2}$ G. M. Sheldrick ${ }^{1}$

${ }^{1}$ Lehrstuhl fuer Strukturchemie, Georg-August-Universitaet, Goettingen, Germany ${ }^{2}$ Bruker AXS Inc., Madison, USA

Solving the phase problem remains probably, with the exception of crystal growing, the largest bottleneck in structure determination. Although much development occurred in this area over the past 10 years, the search for suitable heavy atom derivatives or selenomethionin expression can be time-consuming and is not applicatory in all cases. The technique of fast incorporation of anomalous scatterers in the form of anions and cations, pioneered by Zbigniew Dauter, seems to be widely applicable and relatively fast. Its combination with the use of in-house sources offers an alternative to other, well-established phasing techniques. The steps from preparation to initial map will be discussed with the emphasis on iodide soaking. The iodide anomalous signal at $\mathrm{Cu} \mathrm{K \alpha}$ wavelength is easily measurable and gives instant diagnostics of successful incorporation. However, the subsequent steps in phasing are inherently complicated due to the non-specific nature of binding. The more common pitfalls will be presented, such as the handling of a large number of sites with variable occupancy. Differences between soaking and co-crystallization will be illustrated and recipes suggested for data collection, heavy atom substructure solution with SHELXD, phasing and phase improvement with SHELXE.

Keywords: SAD PHASING IODIDE SOAKS SHELX

Acta Cryst. (2002). A58 (Supplement), C82

X-RAY EXTENDED-RANGE TECHNIQUE FOR PRECISION MEASUREMENT OF THE X-RAY MASS ATTENUATION COEFFICIENT AND IM(F) FOR MOLYBDENUM USING SYNCHROTRON RADIATION

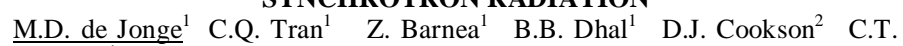
Chantler $^{1}$

${ }^{1}$ University of Melbourne School of Physics University of Melbourne PARKVILLE VICTORIA 3010 AUSTRALIA ${ }^{2}$ ANSTO, Private Mail Bag 1, Menai, NSW 2234 Australia; \& Chem-Mat-CARS-CAT, Argonne National Laboratory

Complex X-Ray form factors are used in crystallography, material science, medical diagnosis, refractive index studies and XAFS. Determinations of the complex component typically differ by around $10 \%$ or 10 standard deviations. This has long been a concern of the International Union of Crystallography, and we have seriously undertaken to determine sources of systematic error that have plagued these measurements. We introduce the X-Ray Extended-Range Technique for accurate measurements of the mass attenuation coefficient and the imaginary component of the atomic form factor. We present our latest experimental results for Molybdenum which feature an absolutely calibrated energy scale, harmonic component determination and correction at a level of 1 photon in 104, sample thickness calibration (by numerous independent methods including weight/area analysis, micrometry and X-Ray scanning). The range of the attenuation measurement far exceeds the Nordfors range of 2 $<\ln (\mathrm{Io} / \mathrm{I})<4$, resulting in an increase of precision and accuracy to below 0.3 $\%$ in the range from $13.5 \mathrm{keV}$ to $41 \mathrm{keV}$. The new result challenges available theoretical calculations and suggests that new methods of computation are required to approach the accuracy of the experimental data, while challenging us to develop a theory of XAFS capable of understanding the absolute magnitude of the fine structure oscillations.

Keywords: FORM FACTORS EXAFS ATTENUATION 